\title{
MS11-P01 | NEW ARP/WARP WEB SERVICE INTEGRATED INTO VIRTUAL COMPUTATION
}

\section{FRAMEWORKS}

Sobolev, Egor (EMBL, Hamburg Unit, Hamburg, GER); Heuser, Philipp (DESY, Hamburg, GER); Chojnowski, Grzegorz (EMBL, Hamburg Unit, Hamburg, GER); Oezugurel, Umut (EMBL, Hamburg Unit, Hamburg, GER); Kantamneni, Sravya (EMBL, Hamburg Unit, Hamburg, GER); Lamzin, Victor (European Molecular Biology Laboratory Hamburg, GER)

The remote web service for macromolecular model building using ARP/WARP [1] was established in 2004 and has had over 5,000 users. Since 2017 it has undergone a complete redesign and now offers the use of all modules of the latest ARP/WARP software version 8.0 for crystallographic model building, including interpretation of cryoelectron microscopy maps. The new web service includes advanced options for real-time monitoring, re-running computational tasks with modified parameters and a comparison of the results.

The redesigned web service is gaining popularity: 900 remote users ran 9,400 model-building tasks in 2018. $92 \%$ of the tasks follow a molecular replacement-like scenario where some initial model is already available. Recent developments implemented in ARP/wARP enable to build atomic structures not only in maps of a medium-to-high resolution but also within 3.0-4.0 ̊̊ resolution range.

Half of the users operate Mac and Linux computers and 30\% use Windows. A considerable portion of users (20\%) submit or monitor tasks from tablets and smartphones.

The ARP/wARP web service is gradually integrated into virtual frameworks [2]. Most of the tasks (58\%) are submitted via the dedicated web interface. Other model-building tasks are submitted to the web service automatically from the CCP4i interface and the molecular replacement pipelines Balbes, MoRDa and MrBump. The users also have an option to direct the built ARP/wARP model to PDB-REDO.

[1] Langer et al., (2008) Nature Protocols. 3, 1171-1179.

[2] Morris et al., (2019) J Struct Biol. 1, 100006. 